\title{
Supported progressive resistance exercise training to counter the adverse side effects of robot-assisted radical prostatectomy: a randomised controlled trial
}

\author{
Ruth E. Ashton ${ }^{1} \cdot$ Jonathan J. Aning ${ }^{2} \cdot$ Garry A. Tew ${ }^{3} \cdot$ Wendy A Robson $^{4} \cdot$ John M Saxton $^{3}$ (D)
}

Received: 26 June 2020 / Accepted: 13 January 2021 / Published online: 23 January 2021

(C) The Author(s) 2021

\begin{abstract}
Purpose To investigate the effects of a supported home-based progressive resistance exercise training (RET) programme on indices of cardiovascular health, muscular strength and health-related quality of life (HR-QoL) in prostate cancer (PCa) patients after treatment with robot-assisted radical prostatectomy (RARP).

Methods This study was a single-site, two-arm randomised controlled trial, with 40 participants randomised to either the intervention or control group over a 10-month period. In addition to receiving usual care, the intervention group completed three weekly RET sessions using resistance bands for 6 months. Participants performed 3 sets of 12-15 repetitions for each exercise, targeting each major muscle group. The control group received usual care only. Brachial artery flow-mediated dilatation (FMD) was the primary outcome and assessed at baseline, 3 and 6 months. Secondary outcomes included body weight, body fat, aerobic fitness, strength and blood-borne biomarkers associated with cardiometabolic risk.

Results There was no significant difference between the groups in FMD at 3 or 6 months. However, there were improvements in aerobic exercise capacity $(P<0.01)$ and upper- $(P<0.01)$ and lower-limb $(P=0.01)$ strength in favour of the RET group at 6 months, accompanied by greater weight loss $(P=0.04)$ and a reduction in body fat $(P=0.02)$. Improvements in HRQoL were evident in the RET group at 3 and 6 months via the PCa-specific component of the FACT-P questionnaire (both $P<0.01$ ). Five adverse events and one serious adverse event were reported throughout the trial duration.

Conclusion This study demonstrates that home-based RET is an effective and safe mode of exercise that elicits beneficial effects on aerobic exercise capacity, muscular strength and HR-QoL in men who have undergone RARP.

Trial registration ISRCTN10490647.
\end{abstract}

Keywords Prostate cancer $\cdot$ Resistance exercise $\cdot$ Robot-assisted radical prostatectomy $\cdot$ Cardiometabolic

John M Saxton

john.saxton@northumbria.ac.uk

1 Department of Sport, Outdoor and Exercise Science, University of Derby, Derby, UK

2 Bristol Urological Institute, North Bristol NHS Trust, Southmead Hospital, Bristol, UK

3 Department of Sport, Exercise \& Rehabilitation, Faculty of Health \& Life Sciences, University of Northumbria at Newcastle, Room 239, Northumberland Building, Newcastle-upon-Tyne NE1 8ST, UK

4 Department of Urology, Freeman Hospital, Newcastle-upon-Tyne, UK

\section{Introduction}

Robot-assisted radical prostatectomy (RARP) is an established minimally invasive treatment for localised prostate cancer $(\mathrm{PCa})$. Men undergoing RARP are well-counselled about the common side effects of surgery, including erectile dysfunction and urinary incontinence, which can have a negative impact on confidence, masculinity and health-related quality of life (HR-QoL) $[4,5,34]$. Men undergoing surgical treatment for $\mathrm{PCa}$ are often considered to be in good general health, perhaps due to younger age [26] and fewer comorbidities [44]. However, recent cross-sectional data suggest that men treated with RARP an average of 11.7 months 
previously have an approximate one in five chances of a cardiovascular-related event within the next 10 years and a similar risk of suffering from clinically important levels of fatigue [6]. This evidence of elevated cardiovascular risk is consistent with data from 100 consecutive men diagnosed with localised $\mathrm{PCa}$ which showed an intermediate to high Framingham risk score in $>95 \%$ of the patients [17]. Muscular strength may also be adversely affected by surgery in this population. A recent small-scale study reported a reduction in upper- and lower-limb strength, which was accompanied by decreases in upper- and lower-limb lean body mass, following radical prostatectomy [43]. This evidence provides a rationale for developing strategies to improve indices of cardiovascular health and skeletal muscle function in men with localised PCa undergoing RARP.

Current guidance recommends that men with PCa participate in structured exercise programmes to improve their health and HR-QoL $[13,33]$. There is no specific exercise guidance for men after treatment with RARP, other than recommendations for cancer patients experiencing urinary incontinence [25], and this reflects the current lack of evidence for this PCa sub-population [10]. However, a 6-month programme of aerobic (walking) exercise was shown to improve indices of cardiovascular health but not erectile dysfunction after surgical treatment for clinically localised PCa [27]. Furthermore, a 12-week programme of twice-weekly resistance exercise training (RET), which included pelvic floor exercise, improved physical function, continence rate and quality of life versus pelvic floor exercise alone after radical prostatectomy [37].

RET is considered to be safe, and in addition to increasing skeletal muscle mass, it has been shown to be effective for improving indices of cardiovascular health, symptoms of cancer-related fatigue and HRQoL in men with $\mathrm{PCa}$ and older adults $[7,16,34$, 35]. The majority of studies examining the effects of RET in clinical populations and older adults have involved supervised exercise [7]. Because supervised exercise requires travel to an exercise facility, which can be costly and time-consuming for patients, it can act as a barrier to participation. However, a key advantage of RET is that it is a highly accessible form of exercise that patients can perform at home following a short instruction period. Home-based RET can be relatively low-cost and convenient, particularly for older individuals who may have less confidence to use leisure centres and gym facilities [45]. Hence, the main aim of this study was to investigate the effects of a home-based progressive RET programme (encompassing a short period of supervised instruction, followed by remote support) on indices of cardiovascular health in men with PCa undergoing RARP and, secondly, to assess the impact of the exercise programme on muscular strength and HR-QoL.

\section{Methods}

\section{Trial design}

This two-armed randomised controlled trial was designed with patient and public involvement. A panel consisting of 28 patients at different stages of treatment (recruited from PCa clinics and the local PCa support group) and healthcare professionals helped to formulate the study. Furthermore, a patient representative was appointed to the Trial Management Group and was consulted on all aspects of the study.

\section{Participants and setting}

Potentially eligible patients attending outpatient clinics were approached by the urology team and informed about the study. A patient letter and information sheet were provided. Patients keen to participate were contacted by a member of the research team and screened to ensure they met the inclusion criteria of a PCa diagnosis, undergone RARP in the previous 8-12 weeks and completed a cardiopulmonary exercise test (CPET) in the previous 4 months. The study exclusion criteria were receiving any other cancer treatment, planned surgery within the next 3 months and participating in another clinical trial where concurrent participation was deemed inappropriate by a clinical investigator. Consenting men underwent checks of resting heart rate and blood pressure and cardiac function using a 12-lead echocardiogram (ECG). The ECG was conducted at rest and was checked by a medical professional. Participants with no contraindications to exercise were allowed to continue into the study.

All patients were recruited from the Freeman Hospital, Newcastle-upon Tyne. The study received ethical approval from the NHS Research Ethics Committee South Scotland and was prospectively registered on the International Standard Randomised Controlled Trial Number database (ref: ISRCTN10490647). All participants provided written informed consent before enrolment. The Consolidated Standards of Reporting Trials (CONSORT) guidelines were followed to guide the reporting of this study [41].

\section{Randomisation and allocation concealment}

Participants were randomised to either RET or usual care, with both groups receiving standard medical treatment (including pre- and post-operative care). Participants were randomly assigned (1:1) using a computer-generated block randomisation schedule (http://www.randomization.com) 
with a block of size 4 . The block size and allocation sequence were not disclosed to ensure concealment. On completion of screening and the baseline assessment, the lead researcher emailed the randomisation administrator who responded with group allocation (RET or usual care).

\section{Intervention}

The intervention group completed three weekly sessions of RET using resistance bands for 6 months. Participants completed 3 sets of $12-15$ repetitions for each exercise in a circuit [2]. All participants performed 8-10 exercises which targeted the major muscle group such as legs (squat, leg press, quick kicks), abdominals (trunk curl-up, lower abdominal crunch, side bend), back (bent-over row, reverse flies), chest (chest press), shoulders (upright row, lateral raise, front raise) and arms (bicep curl and either elbow extension or elbow kick back). Exercises were performed with 30-60-s interpolated rest intervals until 3 sets of each exercise had been performed. The exercise programme was progressed according to the American College of Sports Medicine (ACSM) guidelines and the OMNI-Resistance Exercise Scale (OMNI-RES) [39]. The OMNI-RES is a perceived exertion scale for resistance exercise that helps to control intensity during strength training exercises and can be applied to both men and women. Participants were instructed either to alter the hand/foot position on the band to increase resistance or to progress onto the next level of resistance band once 7-8 was reached on the OMNI-RES [15]. All participants began on the easiest of the resistance bands for a minimum of 1 week. This enabled all participants to understand the movements involved in each exercise with slow progression of the exercises to help avoid any injury.

\section{Supervision and remote support for the intervention group}

Table 1 describes the level of exercise supervision over the course of the trial. The aim of the supervised sessions was to ensure that each participant received individual face-to-face tuition on the appropriate exercise techniques in a designated room at the hospital. Thereafter, patients were remotely supported by weekly telephone calls lasting approximately 10 min until 3 months. Telephone calls were used to discuss any issues and challenges patients had faced with regard to adherence, exercises and aches and pains, along with advising on exercise progression. After the third month, participants received no further contact with the research team until the 6-month outcome assessment session. An exercise booklet provided instructions for all exercises included in the RET programme and was used to record weekly RET activity levels, including the exercises performed, session duration and frequency and OMNI-RES score, over the 6 months.

\section{Usual care}

The control group received usual care only, comprising an outpatient follow-up appointment every 3 months and advice regarding incontinence. They were instructed to continue with their usual activity levels, but no exercise guidance was provided. Usual care for both groups was not affected by this trial.

\section{Outcome measures}

All outcomes were assessed at baseline (prior to randomisation) and at 3- and 6-month follow-up. In addition to the primary and secondary outcomes, participant demographic characteristics such as age, ethnicity, time since surgery, comorbidities and pre-operative prostate-specific antigen (PSA) level were recorded at baseline.

\section{Primary outcome}

Flow-mediated dilatation (FMD) was the primary outcome. FMD is predictive of future cardiovascular events [46], and there is evidence that men treated with RARP are at elevated risk $[6,17]$. FMD assesses the ability of the brachial artery to dilate in response to shear stress to test endothelial function. Participants laid in the supine position for $5 \mathrm{~min}$ prior to the test. A manual sphygmomanometer was placed distal to the olecranon process, and a resting measurement of vessel
Table 1 Level of supervision received by the RET group

\begin{tabular}{ll}
\hline Week & Supervision \\
\hline 1 & 3 supervised exercise sessions \\
2 & 2 supervised sessions and 1 in the home environment (i.e. unsupervised) \\
$3-4$ & 1 supervised session and 2 completed at home \\
$5-12$ & $\begin{array}{l}\text { All exercise sessions were unsupervised, but participants in the intervention group received weekly } \\
\text { telephone contact from a member of the research team [23] }\end{array}$ \\
$13-24$ & All sessions were unsupervised with no contact from the research team \\
\hline
\end{tabular}

N.B. Those who did not take up the invite of supervised sessions were contacted weekly via telephone from week 1 
diameter was performed for $1 \mathrm{~min}$ before cuff inflation to a pressure $50 \mathrm{mmHg}$ above systolic blood pressure (SBP). Occlusion was maintained for $5 \mathrm{~min}$. Recordings were restarted $30 \mathrm{~s}$ before cuff release and continued for a further 3 min thereafter [46]. FMD measurements were completed at each assessment visit. Measurements of the artery diameter were taken, along with measures of shear rate. Analysis of FMD recordings was undertaken using Cardiovascular Suite software (Quipu v3.4, 2018). Measurements of baseline diameter $(\mathrm{mm})$, maximum diameter $(\mathrm{mm})$, recovery diameter $(\mathrm{mm})$, baseline shear rate $\left(\mathrm{s}^{-1}\right)$, maximum shear rate $\left(\mathrm{s}^{-1}\right)$, area to maximum and FMD (\%) were all recorded.

\section{Secondary outcome measures}

Participants arrived fasted for venous blood sample collection which were analysed for blood lipid, insulin and glucose levels. Anthropometric variables, associated with cardiometabolic risk, were also assessed in the fasted state, including body mass index (BMI), waist circumference and skinfold analysis (triceps, subscapular, biceps, iliac crest, supra-spinal, abdominal, front thigh, medial calf). Exercises from the senior fitness test were used to evaluate upper- and lower-limb strength [40]. Lower-limb strength was assessed using the chair sit-to-stand test, and upper-limb strength was assessed using the bicep curl test, requiring men to repeatedly lift an $8 \mathrm{lb}$. (3.63 kg) weight for $30 \mathrm{~s}$ [40].

Submaximal aerobic capacity was assessed using the Bruce ramp protocol on a motorised treadmill (Life Fitness, Next Gen 9500 Treadmill, Cambridge, UK) [39]. The exercise test was terminated when the participant reached 15 on Borg's 620 Rating of Perceived Exertion Scale [28] or for safety reasons if the participant demonstrated an abnormal response to exercise (e.g. very high heart rate, chest pain, light headedness). $\dot{\mathrm{VO}}_{2}$ peak was estimated from the level reached by the participant and using the ACSM Guidelines for Exercise Testing and Prescription [39].

Questionnaires were used to assess QoL (EQ-5D-5L [48] and FACT-P [19]), fatigue (Brief Fatigue Inventory [31]) and self-reported exercise behaviour (modified Godin Leisure Time Exercise Questionnaire [3]). All permissions were sought prior to questionnaire use.

\section{Blinding}

Due to the nature of the intervention, participants were not blinded to group allocation. Assessments at 3 and 6 months that could be influenced by the researcher (e.g. aerobic capacity) were conducted by a trained research assistant blinded to group allocation to minimise the risk of bias. Analysis of blood samples and FMD data were also conducted blindly. Questionnaires were completed by participants independently and checked by a researcher for completeness.

\section{Sample size estimation}

The trial was powered to detect a $2.2 \%$ absolute difference between groups in FMD at 3 months. Previous studies indicate that this is a clinically meaningful difference in terms of future cardiometabolic risk $[8,51]$ and one that is realistic to expect in a parallel-group study in which one group receive a 3 -month structured exercise programme [22]. Assuming a standard deviation of $2.8 \%$ [21], the anticipated effect size was approximately 0.80 . The power calculation indicated that to observe a difference of this magnitude with $80 \%$ power and $5 \%$ two-sided significance, a total sample size of 52 participants was required. Allowing for $15 \%$ attrition, we aimed to recruit 60 participants to the trial, with 30 participants randomised to each group.

\section{Data analysis and missing data}

Normality of distribution for the outcome measures was tested using the Shapiro Wilks test, and assumptions were tested prior to analysis. The effect of the intervention was evaluated using an analysis of covariance (ANCOVA) model, with the baseline value of the outcome included as the covariate. The treatment effect (intervention minus control) is presented with 95\% confidence intervals (CI). Multiple imputation was used for missing data prior to an intention-to-treat analysis being conducted [30]. There were missing data for some variables, and further details are provided in the supplementary material. Analyses run on the dataset were pooled according to Rubin's (1987) rules. All analyses were conducted using IBM SPSS Statistics Version 22 (IBM United Kingdom Limited, Hampshire, UK).

\section{Results}

\section{Participants}

A CONSORT diagram illustrating recruitment, randomisation and completion is presented in the supplementary material. Of 73 eligible participants, 42 men were recruited to the trial and randomised over a 10-month period from June 2017 to April 2018. Participant demographic information is presented in Table 2.

\section{Intervention adherence}

The RET group completed 8-10 exercises per session, with each session lasting 20-30 $\mathrm{min}$. Adherence to the RET intervention in the first 3 months was $94.1 \% \pm 10.5 \%$ with a mean of $1.9 \pm 3.8$ sessions missed. During the first 3 months, one participant suffered a pulmonary embolism judged to be unrelated to the study, and this patient was unable to complete 
Table 2 Participant demographics at baseline

\begin{tabular}{lll}
\hline & $\begin{array}{l}\text { Exercise group } \\
(n=20)\end{array}$ & $\begin{array}{l}\text { Usual care } \\
(n=22)\end{array}$ \\
\hline Age (years) & $64.6 \pm 6.2$ & $66.9 \pm 6.8$ \\
Stature $(\mathrm{cm})$ & $176.9 \pm 7.8$ & $175.8 \pm 6.5$ \\
Body mass $(\mathrm{kg})$ & $88.0 \pm 13.3$ & $87.6 \pm 13.9$ \\
Body mass index $\left(\mathrm{kg} / \mathrm{m}^{2}\right)$ & $28.1 \pm 3.5$ & $28.3 \pm 4.1$ \\
White British $n(\%)$ & $19(95 \%)$ & $22(100 \%)$ \\
Weeks since surgery & $10 \pm 1$ & $11 \pm 2$ \\
Pre-operative PSA $(\mathrm{ng} / \mathrm{ml})$ & $12.1 \pm 10.9$ & $11.9 \pm 11.3$ \\
Hypertension $n(\%)$ & $14(33.3 \%)$ & $8(19.0 \%)$ \\
Hypercholesterolemia $n(\%)$ & $6(14.3 \%)$ & $9(21.4 \%)$ \\
T2DM $n(\%)$ & $4(9.5 \%)$ & $1(2.4 \%)$ \\
Asthma $n(\%)$ & $4(9.5 \%)$ & $0(0 \%)$ \\
Atrial fibrillation $n(\%)$ & $2(4.8 \%)$ & $0(0 \%)$ \\
Arthritis $n(\%)$ & $0(0 \%)$ & $2(4.8 \%)$ \\
Depression $n(\%)$ & $0(0 \%)$ & $2(4.8 \%)$ \\
\hline
\end{tabular}

Data are presented as mean \pm standard deviation unless stated otherwise. PSA prostate-specific antigen, T2DM type 2 diabetes mellitus

2 weeks of the programme as a consequence. In the first 3 months, the three main reasons for missing RET sessions were a bad back, common cold and holidays. In months 36 , adherence in the RET group decreased slightly to $77.7 \%$ $\pm 29.8 \%$, with an average of $8.0 \pm 10.6$ sessions missed. The three main reasons for missing sessions in months 3-6 included becoming a father, Christmas celebrations, a rotator cuff injury and holidays, and three participants did not return their diaries for analysis.

\section{Primary and secondary outcomes}

Baseline data are presented in Tables 3 and 4 . There was no significant difference between the groups for FMD variables at 3 or 6 months (Table 5). However, the RET group experienced improvements in many of the secondary outcomes at the 3- and 6-month follow-ups (Table 5). The adjusted mean difference in submaximal aerobic exercise stage, time and estimated $\dot{V}_{2}$ peak scores were $7.3(3.0,11.6), P<0.01$, $142 \mathrm{~s}(55,232), P<0.01$ and $7.8 \mathrm{ml} / \mathrm{kg} / \mathrm{min}(3.2,12.5), P<$ 0.01 at 3 months respectively and $6.8(2.4,11.1), P<0.01$, $140 \mathrm{~s}(54,226), P<0.01$ and $8.5 \mathrm{ml} / \mathrm{kg} / \mathrm{min}(3.8,13.1), P<$ 0.01 at 6 months, respectively, in favour of the RET group. The adjusted mean upper and lower body strength scores were $3.6(1.7,5.5)$ reps, $P<0.01$ and $3.1(1.0,5.2)$ reps, $P<0.01$ at 3 months, respectively, and $4.3(1.2,7.3)$ reps, $P<0.01$ and $3.2(0.6,5.9)$ reps, $P=0.02$ at 6 months, respectively, again, in favour of the RET group. In addition, the adjusted mean difference in body mass, percentage fat and sum of 8 skinfolds were $-3.1 \mathrm{~kg}(-6.0,-0.2), P=0.04$,
Table 3 Baseline physiological measures

\begin{tabular}{|c|c|c|}
\hline Outcome & $\begin{array}{l}\text { Exercise group } \\
\text { Mean } \pm \text { SD }\end{array}$ & $\begin{array}{l}\text { Usual care } \\
\text { Mean } \pm \text { SD }\end{array}$ \\
\hline \multicolumn{3}{|l|}{ Flow-mediated dilatation } \\
\hline Baseline diameter (mm) & $4.8 \pm 0.6$ & $4.8 \pm 0.5$ \\
\hline Max diameter (mm) & $5.2 \pm 0.9$ & $5.1 \pm 0.8$ \\
\hline Recovery diameter (mm) & $5.0 \pm 0.6$ & $5.1 \pm 0.4$ \\
\hline $\operatorname{FMD}(\%)$ & $6.9 \pm 2.4$ & $7.6 \pm 2.4$ \\
\hline Shear baseline $\left(\mathrm{s}^{-1}\right)$ & $76.8 \pm 24.5$ & $80.9 \pm 20.8$ \\
\hline Shear max $\left(\mathrm{s}^{-1}\right)$ & $390.8 \pm 76.0$ & $389.8 \pm 143.3$ \\
\hline Shear area to $\max$ & $8558.9 \pm 1638.5$ & $8883.6 \pm 4413.4$ \\
\hline \multicolumn{3}{|l|}{ Cardiovascular health } \\
\hline Resting heart rate (bpm) & $71.7 \pm 12.5$ & $66.8 \pm 9.3$ \\
\hline $\mathrm{SBP}(\mathrm{mmHg})$ & $138 \pm 17$ & $136 \pm 15$ \\
\hline DBP (mmHg) & $82 \pm 13$ & $82 \pm 9$ \\
\hline QRisk-2 Score (\%) & $17.0 \pm 7.7$ & $18.0 \pm 6.8$ \\
\hline \multicolumn{3}{|l|}{ Anthropometric profile } \\
\hline Body mass (kg) & $88.0 \pm 13.3$ & $87.6 \pm 13.9$ \\
\hline Body mass index $\left(\mathrm{kg} / \mathrm{m}^{2}\right)$ & $28.1 \pm 3.5$ & $28.3 \pm 4.1$ \\
\hline Waist circumference $(\mathrm{cm})$ & $99.6 \pm 10.0$ & $103.0 \pm 9.7$ \\
\hline Waist/hip & $1.0 \pm 0.1$ & $1.0 \pm 0.1$ \\
\hline Fat $(\%)$ & $18.3 \pm 4.0$ & $17.9 \pm 4.6$ \\
\hline Sum of 6 skinfolds (mm) & $104 \pm 25$ & $101 \pm 27$ \\
\hline Sum of 8 skinfolds (mm) & $136 \pm 29$ & $133 \pm 35$ \\
\hline \multicolumn{3}{|l|}{ Blood biomarkers } \\
\hline Glucose $(\mathrm{mmol} / \mathrm{L})$ & $6.2 \pm 1.9$ & $5.4 \pm 0.6$ \\
\hline Insulin $(\mu \mathrm{U} / \mathrm{ml})$ & $34.2 \pm 14.7$ & $38.4 \pm 21.5$ \\
\hline HOMA-IR & $2.3 \pm 5.4$ & $0.6 \pm 0.4$ \\
\hline Total Chol (mmol/L) & $5.0 \pm 1.1$ & $5.0 \pm 1.5$ \\
\hline $\mathrm{HDL}(\mathrm{mmol} / \mathrm{L})$ & $1.3 \pm 0.3$ & $1.3 \pm 0.3$ \\
\hline $\mathrm{LDL}(\mathrm{mmol} / \mathrm{L})$ & $3.2 \pm 0.8$ & $2.8 \pm 1.2$ \\
\hline Non-HDL (mmol/L) & $3.7 \pm 1.0$ & $3.8 \pm 1.5$ \\
\hline Triglycerides $(\mathrm{mmol} / \mathrm{L})$ & $1.5 \pm 0.7$ & $1.4 \pm 0.6$ \\
\hline Total/HDL (mmol/L) & $4.0 \pm 0.9$ & $4.0 \pm 1.1$ \\
\hline \multicolumn{3}{|l|}{ Submaximal aerobic exercise } \\
\hline Stage & $30.0 \pm 11.4$ & $29.8 \pm 10.3$ \\
\hline Time (secs) & $580 \pm 229$ & $576 \pm 205$ \\
\hline Estimated $\mathrm{VO}_{2}$ Peak $(\mathrm{ml} / \mathrm{kg} / \mathrm{min})$ & $38.6 \pm 13.8$ & $38.7 \pm 12.5$ \\
\hline Max heart rate (bpm) & $143.2 \pm 17.3$ & $132.9 \pm 17.3$ \\
\hline \multicolumn{3}{|l|}{ Strength } \\
\hline 30-s bicep curl test (reps) & $20.0 \pm 5.7$ & $19.4 \pm 4.2$ \\
\hline 30-s chair stand test (reps) & $17.9 \pm 5.1$ & $16.1 \pm 5.3$ \\
\hline
\end{tabular}

$S D$ standard deviation, $F M D$ flow-mediated dilatation, $S B P$ systolic blood pressure, $D B P$ diastolic blood pressure, HOMA-IR insulin resistance, Chol cholesterol, $H D L$ high-density lipoprotein cholesterol, $L D L$ low-density lipoprotein cholesterol, $\mathrm{VO}_{2} \mathrm{Peak}$ maximal aerobic capacity, reps repetitions, secs seconds

$-1.9 \%(-3.5,-0.4), P=0.02$ and $-13 \mathrm{~mm}(-25,-1), P=$ 0.03 at 6 months, respectively, in favour of the RET group. 
Table 4 Baseline questionnaire measures

\begin{tabular}{|c|c|c|}
\hline Outcome & $\begin{array}{l}\text { Exercise group } \\
\text { Mean } \pm \text { SD }\end{array}$ & $\begin{array}{l}\text { Usual care } \\
\text { Mean } \pm \text { SD }\end{array}$ \\
\hline \multicolumn{3}{|l|}{ Quality of life } \\
\hline EQ-5D Index Score & $0.9 \pm 0.1$ & $0.8 \pm 0.2$ \\
\hline ED-5D VAS & $82 \pm 13$ & $78 \pm 14$ \\
\hline \multicolumn{3}{|l|}{ FACT-P } \\
\hline Physical WB & $25.4 \pm 1.8$ & $24.3 \pm 2.9$ \\
\hline Social WB & $23.4 \pm 3.5$ & $22.5 \pm 3.6$ \\
\hline Emotional WB & $21.6 \pm 2.4$ & $20.8 \pm 3.7$ \\
\hline Functional WB & $21.5 \pm 5.2$ & $21.2 \pm 5.5$ \\
\hline Prostate cancer specific & $34.5 \pm 3.2$ & $33.3 \pm 5.2$ \\
\hline FACT-P TOI & $81.4 \pm 8.7$ & $78.8 \pm 11.5$ \\
\hline FACT-P Total Score & $126.4 \pm 12.1$ & $122.2 \pm 16.2$ \\
\hline FACT-G Total Score & $91.9 \pm 10.0$ & $88.8 \pm 12.1$ \\
\hline \multicolumn{3}{|l|}{ Fatigue } \\
\hline BFI & $1.2 \pm 1.2$ & $1.9 \pm 1.4$ \\
\hline \multicolumn{3}{|c|}{ Godin Leisure Time Exercise Questionnaire (modified) } \\
\hline VA frequency (days/week) & $0.0 \pm 0.0$ & $0.4 \pm 1.5$ \\
\hline VA duration (mins/session) & $0.0 \pm 0.0$ & $1.1 \pm 3.8$ \\
\hline MIA frequency (days/week) & $1.8 \pm 2.8$ & $1.1 \pm 2.0$ \\
\hline MIA duration (mins/session) & $15.9 \pm 27.6$ & $25.7 \pm 56.5$ \\
\hline LIA frequency (days/week) & $4.6 \pm 2.2$ & $5.3 \pm 2.6$ \\
\hline LIA duration (mins/session) & $40.0 \pm 31.5$ & $59.6 \pm 61.4$ \\
\hline RET frequency (days/week) & $0.4 \pm 1.6$ & $0.5 \pm 1.6$ \\
\hline RET duration (mins/session) & $0.5 \pm 2.2$ & $2.5 \pm 9.7$ \\
\hline
\end{tabular}

$S D$ standard deviation, $V A S$ visual analogue scale, $W B$ well-being, $T O I$ trial outcome index, $V A$ vigorous aerobic activity, MIA moderate intensity aerobic activity, LIA low-intensity aerobic activity, RET resistance exercise training

However, there were no significant differences between the groups in resting blood pressure, resting heart rate or blood biomarkers.

There were no changes in EQ-5D or BFI scores at 3 or 6 months (Table 6). However, there were significant changes in some aspects of the FACT-P. The adjusted mean difference in functional well-being, PCa specific and FACT-P Trial Outcome Index (TOI) were $1.9(0.3,3.5), P=0.02,2.3$ (1.0, $3.7), P<0.01$ and $5.0(1.9,9.1), P<0.01$ at 3 months, respectively, and $1.9(0.01,3.8), P=0.04,3.1(1.3,5.0), P<0.01$ and $5.0(1.8,8.3), P<0.01$ at 6 months, respectively, in favour of the RET group. Significant changes were also evident for moderate-intensity activity at 3 months $(22.1$ [4.1, 40.0], $P=$ $0.02)$.

\section{Missing data}

Approximately $38 \%$ of participants had some missing data over the 6 months of follow-up for FMD. All participants who attended follow-up sessions had blood taken; however, approximately $15 \%$ of participants did not have some sample results returned from the laboratory or the results were incomplete. The main reason for missing data points was participant drop-out.

\section{Adverse events}

Five adverse events (hernia, accident resulting in back pain, abdominal pain, increased fasted insulin level, rotator cuff injury) and one serious adverse event (pulmonary embolism) were reported throughout the trial duration. Only the report of a rotator cuff injury was related to the trial and was the result of a participant choosing to progress to a more difficult resistance band despite being advised otherwise.

\section{Discussion}

This is the first study to explore the health benefits of a predominantly home-supported progressive RET programme with remote supervision in PCa patients who have recently undergone RARP. The study demonstrates that RET can be implemented safely 8 weeks after RARP and can lead to improvements in a range of important health outcomes. Furthermore, there was a high level of adherence to homebased RET throughout the whole 6 months of the programme.

The results provide some evidence of improved cardiovascular health in the group allocated to RET. FMD was used as a simple, non-invasive measure of arterial endothelial function, which has been used previously in studies of exercise training in populations with elevated cardiovascular disease risk, thereby providing clinically relevant data regarding objective risk of CVD [46]. We observed no change in FMD at either the 3month or 6-month time point in the patients allocated to the RET group. However, it is likely that the study was underpowered to detect changes in FMD, as an a priori power calculation indicated that 52 participants were needed to observe statistically significant differences in FMD between the groups. Other studies have reported a change in FMD from $6.2-8.3 \%$ to $9.7-11.8 \%$ after RET programmes, although the participants were young (18-35 years old), pre-hypertensive and healthy young (18 years old) adults, respectively [9, 36]. FMD could be a useful non-invasive technique for detecting changes in arterial function throughout treatment in PCa patients at elevated risk of cardiovascular disease, but as our results were inconclusive, further research is warranted.

This study demonstrated that RET improved aerobic capacity in men post-RARP. This is an important finding given that aerobic exercise capacity is known to decline with increasing age and diagnosed hypertension. Reductions in $\dot{\mathrm{V}}{ }_{2}$ Peak can exacerbate underlying $\mathrm{CV}$ conditions, impair activities of daily living and increase 
Table 5 Cardiometabolic outcome measures

\begin{tabular}{|c|c|c|c|c|}
\hline \multirow[t]{2}{*}{ Outcome } & \multicolumn{2}{|l|}{3 Months } & \multicolumn{2}{|l|}{6 Months } \\
\hline & Adjusted mean diff. between groups $(95 \% \mathrm{CI})$ & $P$ Value & Adjusted mean diff. between groups $(95 \% \mathrm{CI})$ & $P$ Value \\
\hline \multicolumn{5}{|l|}{ Flow-mediated dilatation } \\
\hline Baseline diameter (mm) & $-0.1(-0.4,0.3)$ & 0.4 & $-0.1(-0.4,0.2)$ & 0.4 \\
\hline Max diameter (mm) & $-0.04(-0.4,0.3)$ & 0.7 & $-0.3(-0.7,0.1)$ & 0.2 \\
\hline Recovery diameter (mm) & $0.3(-0.1,0.7)$ & 0.3 & $-0.4(-0.9,0.1)$ & 0.09 \\
\hline $\operatorname{FMD}(\%)$ & $0.2(-0.8,1.2)$ & 0.7 & $-1.6(-4.7,1.4)$ & 0.2 \\
\hline Shear baseline $\left(\mathrm{s}^{-1}\right)$ & $12.5(-27.9,53.0)$ & 0.5 & $-8.2(-61.8,45.4)$ & 0.7 \\
\hline Shear max $\left(\mathrm{s}^{-1}\right)$ & $17.8(-30.1,65.7)$ & 0.3 & $-12.2(-77.0,52.5)$ & 0.3 \\
\hline Shear area to max & $-1874.3(-412.9,5161.5)$ & 0.5 & $-673.1(-3741.5,2395.3)$ & 0.9 \\
\hline \multicolumn{5}{|l|}{ Cardiovascular health } \\
\hline Resting heart rate (bpm) & $10.9(-5,6)$ & 0.7 & $1(-6,7)$ & 0.8 \\
\hline $\mathrm{SBP}(\mathrm{mmHg})$ & $-6(-12,1)$ & 0.07 & $-7(-15,2)$ & 0.1 \\
\hline DBP (mmHg) & $-1(-5,3)$ & 0.6 & $1(-5,7)$ & 0.7 \\
\hline QRisk-2 Score (\%) & $0.1(-2.7,2.9)$ & 0.9 & $0.3(-2.7,3.2)$ & 0.8 \\
\hline \multicolumn{5}{|l|}{ Anthropometric profile } \\
\hline Body mass (kg) & $-1.4(-3.6,0.6)$ & 0.2 & $-3.1(-6.0,-0.2)$ & 0.04 \\
\hline Body mass index $\left(\mathrm{kg} / \mathrm{m}^{2}\right)$ & $-0.4(-1.1,0.2)$ & 0.2 & $-1.0(-1.9,-0.1)$ & 0.03 \\
\hline Waist circumference $(\mathrm{cm})$ & $-1.3(-3.9,1.3)$ & 0.3 & $-2.3(-5.1,0.5)$ & 0.1 \\
\hline Waist/Hip & $0.0(0.0,0.0)$ & 0.6 & $-0.03(-0.1,0.006)$ & 0.09 \\
\hline Fat $(\%)$ & $-13.2(-26.8,0.4)$ & 0.01 & $-1.9(-3.5,-0.4)$ & 0.02 \\
\hline Sum of 6 skinfolds (mm) & $-2(-3,0)$ & 0.05 & $-8(-17,1)$ & 0.07 \\
\hline Sum of 8 skinfolds (mm) & $-12(-26,1)$ & 0.07 & $-13(-25,-1)$ & 0.03 \\
\hline \multicolumn{5}{|l|}{ Blood biomarkers } \\
\hline Glucose $(\mathrm{mmol} / \mathrm{L})$ & $0.03(-1.0,1.0)$ & 0.9 & $-0.3(-1.1,0.4)$ & 0.4 \\
\hline Insulin $(\mu \mathrm{U} / \mathrm{ml})$ & $-15.2(-46.2,15.9)$ & 0.3 & $-10.7(-46.7,25.2)$ & 0.5 \\
\hline HOMA-IR & $-0.3(-1.0,0.3)$ & 0.3 & $-0.3(-0.9,0.3)$ & 0.3 \\
\hline Total Chol (mmol/L) & $-0.1(-0.6,0.4)$ & 0.6 & $-0.4(-0.9,0.03)$ & 0.07 \\
\hline $\mathrm{HDL}(\mathrm{mmol} / \mathrm{L})$ & $-0.04(-0.5,0.4)$ & 0.6 & $0.01(-0.1,0.1)$ & 0.08 \\
\hline $\mathrm{LDL}(\mathrm{mmol} / \mathrm{L})$ & $-0.2(-0.8,0.4)$ & 0.8 & $-0.3(-0.7,0.1)$ & 0.2 \\
\hline Non-HDL (mmol/L) & $-0.2(-0.5,0.003)$ & 0.5 & $-0.3(-0.9,0.2)$ & 0.2 \\
\hline Triglycerides (mmol/L) & $0.1(-0.6,0.8)$ & 0.7 & $-0.2(-0.6,0.2)$ & 0.4 \\
\hline Total/HDL (mmol/L) & $0.01(-0.5,0.6)$ & 0.9 & $-0.2(-0.7,0.3)$ & 0.4 \\
\hline \multicolumn{5}{|l|}{ Submaximal aerobic exercise } \\
\hline Stage & $7.3(3.0,11.6)$ & $<0.01$ & $6.8(2.4,11.1)$ & $<0.01$ \\
\hline Time (secs) & $142(55,232)$ & $<0.01$ & $140(54,226)$ & $<0.01$ \\
\hline Estimated $\mathrm{VO}_{2}$ Peak (ml/kg/min) & $7.8(3.2,12.5)$ & $<0.01$ & $8.5(3.8,13.1)$ & $<0.01$ \\
\hline Max heart rate (bpm) & $3.1(-2.8,9.1)$ & 0.2 & $6.8(-0.8,14.4)$ & 0.07 \\
\hline \multicolumn{5}{|l|}{ Strength } \\
\hline 30-s bicep curl test (reps) & $3.6(1.7,5.5)$ & $<0.01$ & $4.3(1.2,7.3)$ & $<0.01$ \\
\hline 30-s chair stand test (reps) & $3.1(1.0,5.2)$ & $<0.01$ & $3.2(0.6,5.9)$ & 0.01 \\
\hline
\end{tabular}

$C I$ confidence interval, FMD flow-mediated dilatation, SBP systolic blood pressure, DBP diastolic blood pressure, HOMA-IR insulin resistance, Chol cholesterol, $H D L$ high-density lipoprotein cholesterol, $L D L$ low-density lipoprotein cholesterol, $\mathrm{VO}_{2}$ Peak maximal aerobic capacity, reps repetitions, secs seconds. Statistical significance: $P 0.05$

the risk of depression and functional dependence [14, 47]. Submaximal exercise testing, as employed in this study, is a means of predicting $\mathrm{VO}_{2}$ Peak without performing maximal exercise and may be associated with fewer risks for clinical populations. This type of testing could allow clinicians to monitor patient progress and be used as a 
Table 6 Questionnaire-based outcome measures

\begin{tabular}{|c|c|c|c|c|}
\hline \multirow[t]{2}{*}{ Outcome } & \multicolumn{2}{|l|}{3 Months } & \multicolumn{2}{|l|}{6 Months } \\
\hline & $\begin{array}{l}\text { Adjusted mean diff. between } \\
\text { groups }(95 \% \mathrm{CI})\end{array}$ & $\begin{array}{l}P \\
\text { Value }\end{array}$ & $\begin{array}{l}\text { Adjusted mean diff. between } \\
\text { groups }(95 \% \mathrm{CI})\end{array}$ & $\begin{array}{l}P \\
\text { Value }\end{array}$ \\
\hline \multicolumn{5}{|l|}{ Quality of life } \\
\hline EQ-5D Index Score & $0.02(-0.04,0.1)$ & 0.6 & $0.1(-0.01,0.1)$ & 0.1 \\
\hline ED-5D VAS & $1(-4,7)$ & 0.7 & $-1(-6,4)$ & 0.7 \\
\hline \multicolumn{5}{|l|}{ FACT-P } \\
\hline Physical WB & $0.8(-0.4,2.0)$ & 0.2 & $0.2(-0.9,1.3)$ & 0.8 \\
\hline Social WB & $1.2(-0.4,2.8)$ & 0.1 & $-2.4(-8.3,3.5)$ & 0.4 \\
\hline Emotional WB & $-0.7(-1.9,0.6)$ & 0.3 & $1.1(-0.1,2.3)$ & 0.08 \\
\hline Functional WB & $1.9(0.3,3.5)$ & 0.02 & $1.9(0.01,3.8)$ & 0.04 \\
\hline $\begin{array}{l}\text { Prostate cancer } \\
\text { specific }\end{array}$ & $2.3(1.0,3.7)$ & $<0.01$ & $3.1(1.3,5.0)$ & $<0.01$ \\
\hline FACT-P TOI & $5.0(1.9,9.1)$ & $<0.01$ & $5.0(1.8,8.3)$ & $<0.01$ \\
\hline $\begin{array}{l}\text { FACT-P Total } \\
\text { Score }\end{array}$ & $5.3(0.7,9.8)$ & 0.03 & $4.2(-1.6,10.1)$ & 0.2 \\
\hline $\begin{array}{l}\text { FACT-G Total } \\
\text { Score }\end{array}$ & $3.1(-0.8,7.0)$ & 0.1 & $0.9(-4.0,5.7)$ & 0.7 \\
\hline \multicolumn{5}{|l|}{ Fatigue } \\
\hline BFI & $-0.5(-1.3,0.3)$ & 0.3 & $-0.1(-0.9,0.6)$ & 0.7 \\
\hline \multicolumn{5}{|c|}{ Godin Leisure Time Exercise Questionnaire (modified) } \\
\hline $\begin{array}{r}\text { VA frequency } \\
\text { (days/week) }\end{array}$ & $0.1(-0.4,0.7)$ & 0.7 & $0.4(-0.1,0.9)$ & 0.2 \\
\hline $\begin{array}{l}\text { VA duration } \\
\text { (mins/session) }\end{array}$ & $-27.2(-66.3,11.9)$ & 0.2 & $-3.2(-14.2,7.8)$ & 0.6 \\
\hline $\begin{array}{l}\text { MIA frequency } \\
\text { (days/week) }\end{array}$ & $0.2(-1.2,1.6)$ & 0.8 & $0.3(-0.9,1.5)$ & 0.6 \\
\hline $\begin{array}{l}\text { MIA duration } \\
\text { (mins/session) }\end{array}$ & $22.1(4.1,40.0)$ & 0.02 & $16.9(-8.1,42.0)$ & 0.2 \\
\hline $\begin{array}{l}\text { LIA frequency } \\
\text { (days/week) }\end{array}$ & $-1.0(-2.4,0.4)$ & 0.2 & $-0.7(-2.2,0.8)$ & 0.4 \\
\hline $\begin{array}{l}\text { LIA duration } \\
\text { (mins/session) }\end{array}$ & $-10.4(-33.9,13.2)$ & 0.4 & $3.4(-24.1,30.9)$ & 0.8 \\
\hline $\begin{array}{l}\text { RET frequency } \\
\text { (days/week) }\end{array}$ & $2.6(1.8,3.5)$ & $<0.01$ & $1.9(1.0,2.9)$ & $<0.01$ \\
\hline $\begin{array}{l}\text { RET duration } \\
\text { (mins/session) }\end{array}$ & $27.8(20.7,35.0)$ & $<0.01$ & $17.4(10.8,24.0)$ & $<0.01$ \\
\hline
\end{tabular}

$C I$ confidence interval, $V A S$ visual analogue scale, $W B$ well-being, $T O I$ trial outcome index, $V A$ vigorous aerobic activity, MIA moderate intensity aerobic activity, LIA low-intensity aerobic activity, RET resistance exercise training. Statistical significance: $P<0.05$ tool to motivate men in continuing with an exercise programme.

There were favourable but non-significant reductions in SBP at the 3- and 6-month follow-up time-points in the RET group. Approximately $50 \%$ of myocardial infarctions and strokes in the UK are attributed to hypertension, and so interventions to reduce SBP amongst men treated for PCa could contribute to a reduction in future cardiovascular events [11]. Importantly, hypertension was shown to be significantly more prevalent in a cohort of 100 consecutive men diagnosed with localised PCa [17], and CVD has been identified as one of the leading causes of death in men following RARP [38, 42]. Our results suggest that RET could be used as a highly accessible, non-pharmacological method of reducing arterial blood pressure in men with localised PCa treated with RARP. The design of RET programme used in this study also enables implementation during the current COVID-19 pandemic. Home-based programmes with remote support (via telephone, videoconferencing, etc.) prevent the need for patients to travel to exercise venues and allow for social distancing measures to be heeded. However, further research would be needed to determine the effectiveness of the initial instruction sessions if carried out virtually. 
Improvements were observed in both upper- and lowerlimb muscular strength in the RET group compared with usual care. As the average age of those recruited in this study was $>60$ years, this suggests that home-based RET could help to prevent or ameliorate the age-related loss of skeletal muscle mass and function (i.e. myopenia, sarcopenia, etc.) [2]. Improvements in upper- and lower-limb strength have previously been reported in studies of $\mathrm{PCa}$ after programmes of RET [20, 50]. However, aside from these improvements in skeletal muscle strength and function, there were significant changes in all body composition variables in favour of the RET group over 6 months of follow-up, suggesting RET is beneficial for promoting fat loss in PCa patients undergoing RARP, and this is consistent with data from other populations [49]. The effects of RET on body fat stores are potentially attributable to an increase in total daily energy expenditure on exercising days and as the involved skeletal muscles adapt to training, as some studies have shown increases in basal metabolic rates and fat oxidation following RET [1,29].

Finally, the results of this study suggest that RET can improve patient-reported functional well-being and HR-QoL. This is noteworthy, given that studies have recently reported mental health issues and reduce HR-QoL in the years immediately after PCa diagnosis [12]. The significant difference between the two groups observed in this study suggests that RET is more beneficial than usual care [18] and could therefore have clinical implications regarding the advice and recommendations clinicians make for patients at this stage of their recovery. Participants reported improved PCa specific side effects via the FACT-P questionnaire. Therefore, it can be concluded that RET did not exert any negative effects on incontinence or erectile dysfunction and that participants in the RET group reported a perceived improvements in these commonly reported side effects of RARP. As a result, men could be counselled by healthcare professionals to not be concerned about the effect RET may have on their functional status especially with the programme being conducted in the home environment. RET did not appear to benefit fatigue but did not worsen reported fatigue levels. The lack of change could be due to the low fatigue levels reported by participants at baseline, and the study was probably underpowered to detect any small, but potentially important, difference in the fatigue outcome. However, other studies have reported that exercise has reduced the levels of fatigue in other cancer populations [32].

\section{Limitations}

This study has a number of important limitations. Firstly, the study was underpowered to detect changes in the primary outcome, though significant beneficial effects were evident for many of the secondary outcomes (e.g. body weight, body fat, aerobic fitness, strength, FACT-P). Secondly, although a widely adopted multiple imputation technique was used to account for missing data points [24], missing data contributes to a loss of statistical power and potential bias. Adding to this, some participants were not fully compliant, with some exercise diaries lost or not returned for analysis. Thirdly, it is possible that due to patients being aware of their participation in an exercise trial, they undertook more daily exercise (in addition to RET), and this may have accounted for the observed increase in moderate-intensity physical activity and improvement in aerobic exercise capacity in this group. Fourthly, diet was not accounted for and may have improved due to participation in the trial, potentially leading to favourable reductions in body/fat mass. Finally, it is unclear how the intervention specifically impacted common side effects of RARP, including erectile dysfunction and urinary incontinence. Future research should expand on these preliminary findings and explore the impact of RET on key health outcomes in a larger multi-centre randomised controlled trial.

\section{Conclusions}

This study highlights that RET is a safe, effective and feasible mode of exercise that elicits cardiometabolic health benefits in men who have undergone RARP. RET is an accessible form of exercise for PCa patients recovering from RARP and, when provided with appropriate information, demonstrates good adherence to the programme and reports few trial-related adverse events. Therefore, clinicians should consider discussing the benefits of exercise, including RET, to patients in the weeks following RARP.

Supplementary Information The online version contains supplementary material available at https://doi.org/10.1007/s00520-021-06002-5.

Acknowledgements Acknowledgement should be given to the Newcastle Upon Tyne Patient and Public Involvement Group for their support and valuable feedback.

Author contributions All authors contributed to the study conception and design. Data collection was performed by Ruth E. Ashton. Analysis and interpretation of the results was performed by Ruth E. Ashton, John M. Saxton, Garry A. Tew and Jonathan J. Aning. The first draft of the manuscript was written by Ruth E. Ashton, and all authors commented on previous versions of the manuscript. All authors read and approved the final manuscript.

Funding This study was funded by The Urology Foundation.

Data availability The research data support our published claims and comply with field standards.

\section{Compliance with ethical standards}

Conflict of interest All authors declare that they have no conflict of interest. 
Ethics approval The study received ethical approval from the South Scotland NHS Research Ethics Committee.

Consent to participate All participants provided informed consent prior to enrolling onto the study.

Consent for publication All authors consent to publication of this article in Supportive Care in Cancer.

Code availability Not applicable.

Open Access This article is licensed under a Creative Commons Attribution 4.0 International License, which permits use, sharing, adaptation, distribution and reproduction in any medium or format, as long as you give appropriate credit to the original author(s) and the source, provide a link to the Creative Commons licence, and indicate if changes were made. The images or other third party material in this article are included in the article's Creative Commons licence, unless indicated otherwise in a credit line to the material. If material is not included in the article's Creative Commons licence and your intended use is not permitted by statutory regulation or exceeds the permitted use, you will need to obtain permission directly from the copyright holder. To view a copy of this licence, visit http://creativecommons.org/licenses/by/4.0/.

\section{References}

1. Alvehus M, Boman N, Soderlund K, Svensson MB, Buren J (2014) Metabolic adaptations in skeletal muscle, adipose tissue, and whole-body oxidative capacity in response to resistance training. Eur J Appl Physiol 114:1463-1471

2. American College of Sports M, Chodzko-Zajko WJ, Proctor DN, Fiatarone Singh MA, Minson CT, Nigg CR, Salem GJ, Skinner JS (2009) American College of Sports Medicine position stand. Exercise and physical activity for older adults. Med Sci Sports Exerc 41:1510-1530

3. Amireault S, Godin G (2015) The Godin-Shephard leisure-time physical activity questionnaire: validity evidence supporting its use for classifying healthy adults into active and insufficiently active categories. Percept Mot Skills 120:604-622

4. Aning JJ, MacKenzie KR, Fabricius M, McColl E, Johnson MI, Tandogdu Z, Soomro NA, Harding C (2018) Detailed analysis of patient-reported lower urinary tract symptoms and effect on quality of life after robotic radical prostatectomy. Urol Oncol 36:364 e315364 e 322

5. Ansmann L, Winter N, Ernstmann N, Heidenreich A, Weissbach L, Herden J (2018) Health-related quality of life in active surveillance and radical prostatectomy for low-risk prostate cancer: a prospective observational study (HAROW - hormonal therapy, active surveillance, radiation, operation, watchful waiting). BJU Int 122:401410

6. Ashton RE, Tew GA, Robson WA, Saxton JM, Aning JJ (2019) Cross-sectional study of patient-reported fatigue, physical activity and cardiovascular status in men after robotic-assisted radical prostatectomy. Support Care Cancer 27:4763-4770

7. Ashton RE, Tew GA, Aning JJ, Gilbert SE, Lewis L, Saxton JM (2020) Effects of short-term, medium-term and long-term resistance exercise training on cardiometabolic health outcomes in adults: systematic review with meta-analysis. Br J Sports Med 54:341-348

8. Atkinson G, Batterham AM (2013) Allometric scaling of diameter change in the original flow-mediated dilation protocol. Atherosclerosis 226:425-427
9. Beck DT, Casey DP, Martin JS, Emerson BD, Braith RW (2013) Exercise training improves endothelial function in young prehypertensives. Exp Biol Med (Maywood, NJ) 238:433-441

10. Bourke L, Smith D, Steed L, Hooper R, Carter A, Catto J, Albertsen PC, Tombal B, Payne HA, Rosario DJ (2016) Exercise for men with prostate cancer: a systematic review and meta-analysis. Eur Urol 69:693-703

11. British Heart Foundation (2020) BHF: UK Factsheet. https://www. bhf.org.uk/what-we-do/our-research/heart-statistics

12. Buffart LM, Newton RU, Chinapaw MJ, Taaffe DR, Spry NA, Denham JW, Joseph DJ, Lamb DS, Brug J, Galvao DA (2015) The effect, moderators, and mediators of resistance and aerobic exercise on health-related quality of life in older long-term survivors of prostate cancer. Cancer 121:2821-2830

13. Campbell KL, Winters-Stone KM, Wiskemann J, May AM, Schwartz AL, Courneya KS, Zucker DS, Matthews CE, Ligibel JA, Gerber LH, Morris GS, Patel AV, Hue TF, Perna FM, Schmitz KH (2019) Exercise guidelines for cancer survivors: consensus statement from International Multidisciplinary Roundtable. Med Sci Sports Exerc 51:2375-2390

14. Chandrasekaran B, Arumugam A, Davis F, Kumaran DS, Chandrasharma B, Khundrakpam C, Chanam L, Philipraj SJ, Sanathombi Devi R (2010) Resistance exercise training for hypertension. Cochrane Database Syst Rev:CD008822

15. Colado JC, Garcia-Masso X, Triplett TN, Flandez J, Borreani S, Tella V (2012) Concurrent validation of the OMNI-resistance exercise scale of perceived exertion with Thera-band resistance bands. J Strength Cond Res 26:3018-3024

16. Cormie P, Galvao DA, Spry N, Joseph D, Chee R, Taaffe DR, Chambers SK, Newton RU (2015) Can supervised exercise prevent treatment toxicity in patients with prostate cancer initiating androgen-deprivation therapy: a randomised controlled trial. BJU Int 115:256-266

17. Davis MK, Rajala JL, Tyldesley S, Pickles T, Virani SA (2015) The prevalence of cardiac risk factors in men with localized prostate cancer undergoing androgen deprivation therapy in British Columbia, Canada. J Oncol 2015:820403

18. Ernstmann N, Weissbach L, Herden J, Winter N, Ansmann L (2017) Patient-physician communication and health-related quality of life of patients with localised prostate cancer undergoing radical prostatectomy - a longitudinal multilevel analysis. BJU Int 119: 396-405

19. Esper P, Mo F, Chodak G, Sinner M, Cella D, Pienta KJ (1997) Measuring quality of life in men with prostate cancer using the functional assessment of cancer therapy-prostate instrument. Urology 50:920-928

20. Galvao DA, Taaffe DR, Spry N, Cormie P, Joseph D, Chambers SK, Chee R, Peddle-McIntyre CJ, Hart NH, Baumann FT, Denham J, Baker M, Newton RU (2018) Exercise preserves physical function in prostate cancer patients with bone metastases. Med Sci Sports Exerc 50:393-399

21. Gilbert SE, Tew GA, Bourke L, Winter EM, Rosario DJ (2013) Assessment of endothelial dysfunction by flow-mediated dilatation in men on long-term androgen deprivation therapy for prostate cancer. Exp Physiol 98:1401-1410

22. Gilbert SE, Tew GA, Fairhurst C, Bourke L, Saxton JM, Winter EM, Rosario DJ (2016) Effects of a lifestyle intervention on endothelial function in men on long-term androgen deprivation therapy for prostate cancer. Br J Cancer 114:401-408

23. Hackshaw-McGeagh LE, Sutton E, Persad R, Aning J, Bahl A, Koupparis A, Millett C, Martin RM, Lane JA (2017) Acceptability of dietary and physical activity lifestyle modification for men following radiotherapy or radical prostatectomy for localised prostate cancer: a qualitative investigation. BMC Urol 17:94 
24. Hayati Rezvan P, Lee KJ, Simpson JA (2015) The rise of multiple imputation: a review of the reporting and implementation of the method in medical research. BMC Med Res Methodol 15:30

25. Hayes SC, Newton RU, Spence RR, Galvao DA (2019) The Exercise and Sports Science Australia position statement: exercise medicine in cancer management. J Sci Med Sport 22:1175-1199

26. Jang TL, Patel N, Faiena I, Radadia KD, Moore DF, Elsamra SE, Singer EA, Stein MN, Eastham JA, Scardino PT, Lin Y, Kim IY, Lu-Yao GL (2018) Comparative effectiveness of radical prostatectomy with adjuvant radiotherapy versus radiotherapy plus androgen deprivation therapy for men with advanced prostate cancer. Cancer 124:4010-4022

27. Jones LW, Hornsby WE, Freedland SJ, Lane A, West MJ, Moul JW, Ferrandino MN, Allen JD, Kenjale AA, Thomas SM, Herndon JE, 2nd, Koontz BF, Chan JM, Khouri MG, Douglas PS, Eves ND (2014) Effects of nonlinear aerobic training on erectile dysfunction and cardiovascular function following radical prostatectomy for clinically localized prostate cancer. Eur Urol 65: 852-855

28. Kaminsky LA, Whaley MH (1998) Evaluation of a new standardized ramp protocol: the BSU/Bruce Ramp protocol. J Cardpulm Rehabil 18:438-444

29. Lemmer JT, Ivey FM, Ryan AS, Martel GF, Hurlbut DE, Metter JE, Fozard JL, Fleg JL, Hurley BF (2001) Effect of strength training on resting metabolic rate and physical activity: age and gender comparisons. Med Sci Sports Exerc 33:532-541

30. Manly CA, Wells RS (2014) Reporting the use of multiple imputation for missing data in higher education research. Res High Educ 56:397-409

31. Mendoza TR, Wang XS, Cleeland CS, Morrissey M, Johnson BA, Wendt JK, Huber SL (1999) The rapid assessment of fatigue severity in cancer patients: use of the Brief Fatigue Inventory. Cancer 85: 1186-1196

32. Meneses-Echavez JF, Gonzalez-Jimenez E, Ramirez-Velez R (2015) Supervised exercise reduces cancer-related fatigue: a systematic review. J Phys 61:3-9

33. National Institute for Health and Care Excellence (2014) Prostate cancer: diagnosis and management. Clinical guideline [CG175] https://www.nice.org.uk/guidance/cg175/ifp/chapter/Treatmentoptions

34. Neil-Sztramko SE, Medysky ME, Campbell KL, Bland KA, Winters-Stone KM (2019) Attention to the principles of exercise training in exercise studies on prostate cancer survivors: a systematic review. BMC Cancer 19:321

35. Newton RU, Galvao DA (2008) Exercise in prevention and management of cancer. Curr Treat Options in Oncol 9:135-146

36. Okamoto T, Masuhara M, Ikuta K (2011) Effect of low-intensity resistance training on arterial function. Eur J Appl Physiol 111:743748

37. Park SW, Kim TN, Nam JK, Ha HK, Shin DG, Lee W, Kim MS, Chung MK (2012) Recovery of overall exercise ability, quality of life, and continence after 12-week combined exercise intervention in elderly patients who underwent radical prostatectomy: a randomized controlled study. Urology 80:299-305

38. Public Health England (2017) Health matters: combating high blood pressure https://publichealthmatters.blog.gov.uk/2017/01/ 24/health-matters-combating-high-blood-pressure/
39. Riebe D, Ehrman JK, Liguori G, Magal M, American College of Sports Medicine ib (2017) ACSM's guidelines for exercise testing and prescription. Wolters Kluwer, Philadelphia

40. Rikli RE, Jones CJ (2012) Senior fitness test Maual. Second Edition. Human Kinetics

41. Schulz KF, Altman DG, Moher D, Group C (2010) CONSORT 2010 statement: updated guidelines for reporting parallel group randomised trials. BMJ 340:c332

42. Shikanov S, Kocherginsky M, Shalhav AL, Eggener SE (2012) Cause-specific mortality following radical prostatectomy. Prostate Cancer Prostatic Dis 15:106-110

43. Singh F, Newton RU, Baker MK, Spry NA, Taaffe DR, Thavaseelan J, Galvao DA (2017) Feasibility of presurgical exercise in men with prostate cancer undergoing prostatectomy. Integr Cancer Ther 16:290-299

44. Sooriakumaran P, Nyberg T, Akre O, Haendler L, Heus I, Olsson M, Carlsson S, Roobol MJ, Steineck G, Wiklund P (2014) Comparative effectiveness of radical prostatectomy and radiotherapy in prostate cancer: observational study of mortality outcomes. BMJ 348:g1502

45. Thiebaud RS, Funk MD, Abe T (2014) Home-based resistance training for older adults: a systematic review. Geriatr Gerontol Int 14:750-757

46. Thijssen DH, Black MA, Pyke KE, Padilla J, Atkinson G, Harris RA, Parker B, Widlansky ME, Tschakovsky ME, Green DJ (2011) Assessment of flow-mediated dilation in humans: a methodological and physiological guideline. Am J Phys Heart Circ Phys 300:H2H12

47. Tucker WJ, Lijauco CC, Hearon CM Jr, Angadi SS, Nelson MD, Sarma S, Nanayakkara S, La Gerche A, Haykowsky MJ (2018) Mechanisms of the improvement in peak VO2 with exercise training in heart failure with reduced or preserved ejection fraction. Heart Lung Circ 27:9-21

48. Van Reenan M, \& Janssen, B (2015) EQ-5D-5L user guide: basic information on how to use the EQ5D-5L instrument. Version 2.1 https://euroqol.org

49. Willis LH, Slentz CA, Bateman LA, Shields AT, Piner LW, Bales CW, Houmard JA, Kraus WE (2012) Effects of aerobic and/or resistance training on body mass and fat mass in overweight or obese adults. J Appl Physiol (Bethesda, Md : 1985) 113:1831-1837

50. Winters-Stone KM, Lyons KS, Dobek J, Dieckmann NF, Bennett JA, Nail L, Beer TM (2016) Benefits of partnered strength training for prostate cancer survivors and spouses: results from a randomized controlled trial of the Exercising Together project. J Cancer Surviv 10:633-644

51. Yeboah J, Folsom AR, Burke GL, Johnson C, Polak JF, Post W, Lima JA, Crouse JR, Herrington DM (2009) Predictive value of brachial flow-mediated dilation for incident cardiovascular events in a population-based study: the multi-ethnic study of atherosclerosis. Circulation 120:502-509

Publisher's note Springer Nature remains neutral with regard to jurisdictional claims in published maps and institutional affiliations. 